\title{
Early hearing screening in South Africa - time to get real about context
}

Review of published literature related to early hearing detection and intervention (EHDI) services in South Africa (SA) between 1995 and 2014 has highlighted progress in terms of the journey toward implementation of paediatric hearing screening services. ${ }^{[1]}$ However, in contrast to developed contexts, there are very few outcome studies from developing countries such as SA that support the efficacy of EHDI. This dearth of evidence from developing countries may be due to the lack of integrated, national EHDI programmes.

The Health Professions Council of South Africa (HPCSA) ${ }^{[2]}$ position statement guidelines and principles for EHDI are primarily based on guidelines from developed contexts with slight contextual adaptations in terms of the time frames for screening and diagnosis. While these guidelines are geared toward universal newborn hearing screening (UNHS) and serve as the gold standard that audiologists in SA should constantly aim towards achieving, they may not necessarily be currently applicable within all healthcare sectors within the SA context. Research and conceptual papers related to EHDI in SA have fortunately acknowledged the impracticalities in attempting to implement developed world models of newborn hearing screening (NHS) in developing countries, where context is evidently different. ${ }^{[1]}$

The current status of $\mathrm{NHS}^{[3]}$ coupled with manpower-related challenges may possibly suggest that UNHS is currently not applicable in the SA context, particularly in the public healthcare sector, where over $80 \%$ of the population receives healthcare. The manpower demand related to a higher prevalence rate of infant hearing loss in the public healthcare sector in SA is not met, as the majority of registered audiologists work in the private healthcare sector. Moreover, there is currently no established mid-level worker programme in audiology to facilitate hearing screening by personnel other than audiologists. There is, therefore, an evident shortage of manpower in the public sector, which may influence the ability of audiologists in SA to effectively implement UNHS. However, it is important that an interim approach to early detection of hearing loss be established as a means of potentially identifying newborns and infants with hearing loss, who would ordinarily be missed in the absence of an NHS programme.

The implementation of risk-based or targeted newborn hearing screening (TNHS) programmes, which involve screening of newborns with known risk factors for hearing loss, would be a more feasible interim approach to adopt in order to identify children with hearing loss early. In SA, TNHS has not been formally and systematically implemented as the intermediate, small step towards a larger UNHS programme. Effective implementation of a TNHS is dependent on a number of aspects, such as: the choice of screening measures, and how these measures are to be employed within a screening protocol; the existence of established risk factors for hearing loss; and the various levels of service delivery or contexts within the SA healthcare system.

High-risk factors for hearing loss stipulated by the HPCSA have been based on position statements from developed contexts, and have been faintly adapted to include two conditions that are considered unique to the SA context. ${ }^{[2]}$ While research findings from developed contexts may be of value, findings from these higher-income countries may be costly and more difficult to implement into practice in lowerincome countries as they may be culturally inappropriate and often focus on non-communicable diseases prevalent in developed contexts. There is therefore a tendency to neglect the specific, local needs of lower-income or developing countries. ${ }^{[4]}$ Hence, contextual research is imperative in guiding clinical and contextually relevant practice. Risk factors for permanent congenital and early-onset hearing loss may also vary across communities and may be influenced by considerable variation of situations, burden of disease and time periods in different countries ${ }^{[5]}$ Context-specific risk factors are particularly relevant to ensure appropriate identification of hearing loss in high-risk neonates. Much ongoing research is therefore needed into the review of highrisk factors for hearing loss within the SA context. Instead of using existing, international high-risk registries for TNHS in SA, it may be more beneficial to screen all neonates with a prolonged hospital stay, whether in neonatal intensive care unit, high care or kangaroo mother care. This will allow for a greater coverage rate and lessen the chances of missed cases in this population. Findings from implemented TNHS programmes could also be used retrospectively to conduct research and consistently update the list of risk indicators associated with hearing impairment.

A variety of healthcare contexts need to be explored as possible platforms for the establishment and provision of NHS services, particularly TNHS as the intermediate approach. This is particularly important as the choice of context may influence coverage rates and follow-up return rates, which are two of the key determinants to effective and successful NHS programmes. Primary healthcare clinics in SA have been proposed as one of the platforms for conducting NHS in order to ensure optimal coverage and follow-up return rate, and this is being piloted in the Western Cape. This was based on statistics in 2002 that indicated a significant number of home births ${ }^{[2]}$ and well babies being discharged soon after birth, making it difficult to co-ordinate screening. However, the number of home births has since decreased, with statistics indicating that $94.1 \%$ of live births occur at a health facility. ${ }^{[6]}$ Hence, more initiatives are required at secondary and tertiary levels of service delivery, particularly in highrisk neonates who may have a prolonged hospital stay and scheduled follow-up appointments with paediatricians at these facilities.

The author is of the view that a two-tiered approach may be appropriate, involving early hearing screening of high-risk babies in the hospital setting, with screening of well babies at clinic level. This would address the earlier discharge of well babies, which would result in missed cases in the hospital setting. It would also address follow-up and monitoring of high-risk infants who undergo regular follow-up at specialist clinics within a hospital setting. Audiologists need to play an active role in the piloting, planning, implementation and management of TNHS programmes within the SA public healthcare context.

\section{Amisha Kanji}

Department of Speech Pathology and Audiology, School of Human and Community Development, University of the Witwatersrand, Johannesburg, South Africa

amisha.kanji@wits.ac.za

1. Moodley S, Storbeck C. Narrative review of EHDI in South Africa. S Afr J Commun Disord 2015;62(1):1-10. http://dx.doi.org/10.4102/sajcd.v62i1.126

2. Health Professions Council of South Africa. Early Hearing Detection and Intervention Programmes in South Africa, Position Statement, Year 2007. http://www.hpcsa.co.za/Uploads/editor/UserFiles/downloads/speech/early_ hearing_detection_statement.pdf (accessed 10 March 2016).

3. Theunissen M, Swanepoel D. Early hearing detection and intervention services in the public health care sector in South Africa. Int J Audiol 2008;47(Suppl 1):S23-29. http://dx.doi.org/10.1080/14992020802294032

4. Chetwood JD, Ladep NG, Taylor-Robinson SD. Research partnerships between high and low-income countries: Are international partnerships always a good thing? BMC Med Ethics 2015;16:36-40. http://dx.doi.org/10.1186/s12910-015-0030-z

5. Olusanya BO, Luxon LM, Wirz SL. Benefits and challenges of newborn hearing screening for developing countries. Int J Pediatr Otorhinolaryngol 2004;68(3):287-305. http://dx.doi.org/10.1016/j.ijporl.2003.10.015

6. South African Government Information. Maternal and Women's Health. 2011. http://www.info.gov.za/aboutsa/health.htm (accessed 19 July 2011). 